\title{
Comparing the accuracy of six intraoral scanners on prepared teeth and effect of scanning sequence
}

\author{
Burcu Diker*, Önjen Tak \\ Department of Prosthodontics, Faculty of Dentistry, Istanbul Okan University, Istanbul, Turkey
}

\begin{abstract}
PURPOSE. The aim of this study was to evaluate the accuracy of six recently introduced intraoral scanners (IOSs) for single crown preparations isolated from the complete arch, and to determine the effect of scanning sequence on accuracy. MATERIALS AND METHODS. A complete arch with right and left canine preparations for single crowns was used as a study model. The reference dataset was obtained by scanning the complete arch using a highly accurate industrial scanner (ATOS Core 80, GOM GmbH). Six different IOSs (Trios, iTero, Planmeca Emerald, Cerec Omnicam, Primescan, and Virtuo Vivo) were used to scan the model ten times each. The scans performed with each IOS were divided into two groups, based on whether the scanning sequence started from the right or left quadrant $(n=5)$. The accuracy of digital impression was evaluated using three-dimensional analyzing software (Geomagic Studio 12, 3D Systems). The Kruskal Wallis and Mann- Whitney U statistical tests for trueness analysis and the One-way ANOVA test for precision analysis were performed $(\alpha=.05)$. RESULTS. The trueness and precision values were the lowest with the Primescan ( 25 and $10 \mu \mathrm{m})$, followed by Trios (40.5 and $11 \mu \mathrm{m})$, Omnicam (41.5 $\mu \mathrm{m}$ and $18 \mu \mathrm{m})$, Virtuo Vivo (52 and $37 \mu \mathrm{m})$, iTero (70 and $12 \mu \mathrm{m})$ and Emerald (73.5 and $60 \mu \mathrm{m})$. Regarding trueness, iTero showed more deviation when scanning started from the right $(P=.009)$.
\end{abstract} CONCLUSION. The accuracy of digital impressions varied depending on the IOS and scanning sequence used. Primescan had the highest accuracy, while Emerald showed the most deviation in accuracy for single crown preparations. [J Adv Prosthodont 2020;12:299-306]

KEYWORDS: Trueness; Precision; Digital impression

\section{INTRODUCTION}

Obtaining accurate impression is a critical step in prosthetic workflow. In addition to conventional impression techniques, digital impressions made with IOSs have been used in clinical practice for over thirty years. Digital impression techniques show clinically acceptable results compared to conventional impression techniques for fixed prosthesis fab-

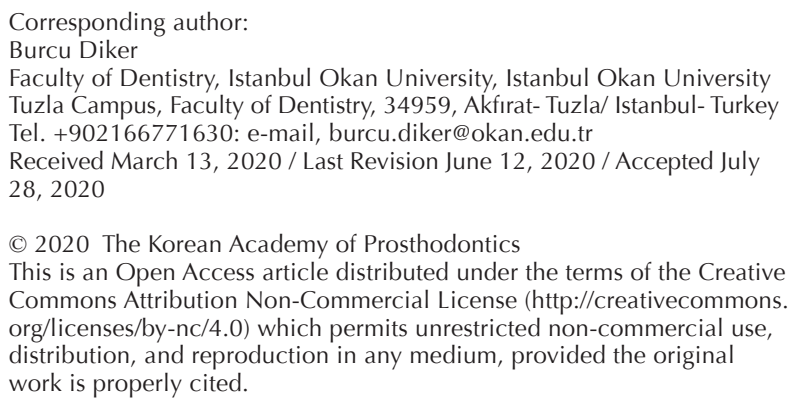

rication. ${ }^{1-3}$ Using digital impression techniques is time efficient $^{4}$, eliminates many clinical and laboratory steps such as selecting the tray, preparing impression material, and pouring a stone model, eliminates potential error due to distortion and deformation of elastic impression materials, ${ }^{5}$ and improves patient acceptance. ${ }^{6}$

The accuracy is specified as trueness and precision according to the International Organization for Standardization. The trueness is defined as 'the closeness between the test object and the reference object' and precision is defined as 'the variability of repeated measurements of the object'. ${ }^{7}$ A scan obtained from a reference scanner is required to evaluate trueness of investigated IOS. In vitro studies ${ }^{8-11}$ commonly use a highly accurate industrial or laboratory scanner as a reference scanner. Three-dimensional comparative analysis software programs using a best-fit alignment method have been used by researchers to evaluate the accuracy of IOSs. ${ }^{12-16}$

Recent technological advances have made digital impressions and crown manufacturing in clinical practice increas- 
ingly common. The chair-side computer-aided design and manufacturing technology utilizing IOSs ensures restoration delivery to the patient within one appointment. ${ }^{17}$ However, the marginal fit of the restoration may vary depending on IOS accuracy. ${ }^{18}$ The acceptable maximum marginal gap was defined as $120 \mu \mathrm{m}$. Data on the effect of digital impression accuracy on the fit of the single crown restoration is lacking in the literature. However, previous studies noted that when evaluating whether the IOS accuracy is within the clinically acceptable range, the digital impression accuracy should be below $120 \mu \mathrm{m} .{ }^{11,16}$ There are few studies in the literature evaluating the accuracy of different IOSs for obtaining digital impression of single crown preparation. ${ }^{8,9,11,18,19}$ The differences in digital impression accuracy were found among IOSs used for single crown preparation. Sim et al. . $^{9}$ reported better accuracy for impression obtained by digital method compared to conventional method for the single crown preparation. However, the results of an in vitro study showed that the conventional workflow was more precise than the digital workflow for transferring the implant position in single implant analog models. ${ }^{20} \mathrm{Kim}$ et al..$^{11}$ demonstrated that accuracy of IOSs differed depending on their data capture principle for a complete arch model, including single crown preparations. Today, there are many IOS systems using various scanning technologies on the market, with and new ones being introduced. In addition to the different scanning technologies used by various manufacturers, new versions of hardware or software may also improve the accuracy of IOSs. ${ }^{12,22}$ To the best of our knowledge, although edentulous or complete arch models were used in the studies evaluating accuracy of digital impression by using Primescan, Virtuo Vivo, and Planmeca Emerald as new introduced scanners, ${ }^{12,13,23,24}$ no study has compared the accuracy of these IOSs for prepared teeth.

Manufacturers describe intraoral scan strategies for single crown preparation, quadrant, and complete arch impression. Nevertheless, the manufacturers have not provided any guidelines about the quadrant in which scanning should begin, especially in cases where both quadrants have prepared teeth. The effect of scanning sequence on digital impression accuracy in the complete arch model was shown in a previous study. ${ }^{25}$ The authors determined a difference in accuracy depending on whether the scans started from the right or left quadrant when using iTero. This difference may be related to using the image stitching process and scan strategy in which the two quadrants are scanned separately and then merged. However, there is a lack of information in the literature as to whether the quadrant where the scan starts affects the accuracy of the digital impression.

The aim of the present study was to evaluate accuracy of six IOSs, and to examine the effect of scanning sequence on digital impression of single crown preparation. The null hypotheses of the study were that (1) there are no differences in accuracy between intraoral scanners; and (2) the scanning sequence does not affect accuracy.

\section{MATERIALS AND METHODS}

A maxillary complete arch model (ANA-4V, Frasaco, Tettnang, Germany) was used in the present study. The right and left canine teeth on the reference model were prepared for single crowns. The preparations were simulated by a $2.0 \mathrm{~mm}$ incisal edge reduction, approximately 10 degree convergence angle, and a $1.0 \mathrm{~mm}$ wide supragingival chamfer margin. All of the sharp angles of the preparations were rounded.

First, the prepared model was scanned using a highly accurate industrial reference scanner (ATOS Core 80, GOM $\mathrm{GmbH}$, Braunschweig, Germany) to create a digital reference dataset. The reference scanner was calibrated and tested. The maximum deviations found were a $1 \mu \mathrm{m}$ probing error form, $3 \mu \mathrm{m}$ probing error size, $5 \mu \mathrm{m}$ sphere spacing error, and $7 \mu \mathrm{m}$ length measurement error. According to the manufacturer's data, this reference scanner uses a stereo camera set-up working on the principle of triangulation. ${ }^{26}$ Six IOSs were used to investigate the precision and trueness of different intraoral scanning systems, as follows: Trios 3 version 1.4.7.5 (3Shape A/S, Copenhagen, Denmark), iTero Element 2 version 1.9.3.3 (Align Technology Inc, San Jose, CA, USA), Cerec Omnicam version 4.6.1 (Dentsply-Sirona Dental Systems, Bensheim, Germany), Planmeca Emerald version 6.0 (Planmeca Oy, Helsinki, Finland), Cerec Primescan version 5.0.0 (Dentsply-Sirona Dental Systems, Bensheim, Germany) and Virtuo Vivo version 3.0 (Dental Wings Inc., Montreal, Canada). One investigator (BD) performed all scans with each intraoral scanner according to the protocols described by each manufacturer. The scan strategies are demonstrated via a representative illustration in Figure 1. Ten scans were taken of the model using each intraoral scanner (a total of 60 scans). The first 5 scans started from the right maxillary quadrant (Scan RightScanR) and the following 5 scans started from the left maxillary quadrant (Scan Left- ScanL) to evaluate the effect of the scanning sequence.

For standardization and subsequent digital processing, datasets from each scan were converted to a standard tessellation language (STL) file format. All of the STL-formatted datasets were loaded into three-dimensional analyzing software (Geomagic Studio 12, 3D Systems, Morrisville, NC, USA) to evaluate the accuracy of the tooth preparations. The left and right canine teeth were digitally isolated from the complete arch to reduce the possible errors that could result from the superposition of large datasets (Fig. 2). ${ }^{16}$ The trimmed left and right canine data was saved as a STLformatted file. The obtained STL data was aligned with the reference STL data while the two canine teeth were connected. For the trueness measurement, these models were superimposed on the reference model using a best-fit algorithm of the software. For the evaluation of precision, 5 scans in the ScanR and ScanL groups were superimposed on each other within groups. A two-way pairwise comparison was performed because reference scan data were not clear in the intra-group comparison. ${ }^{27}$ For example, the 

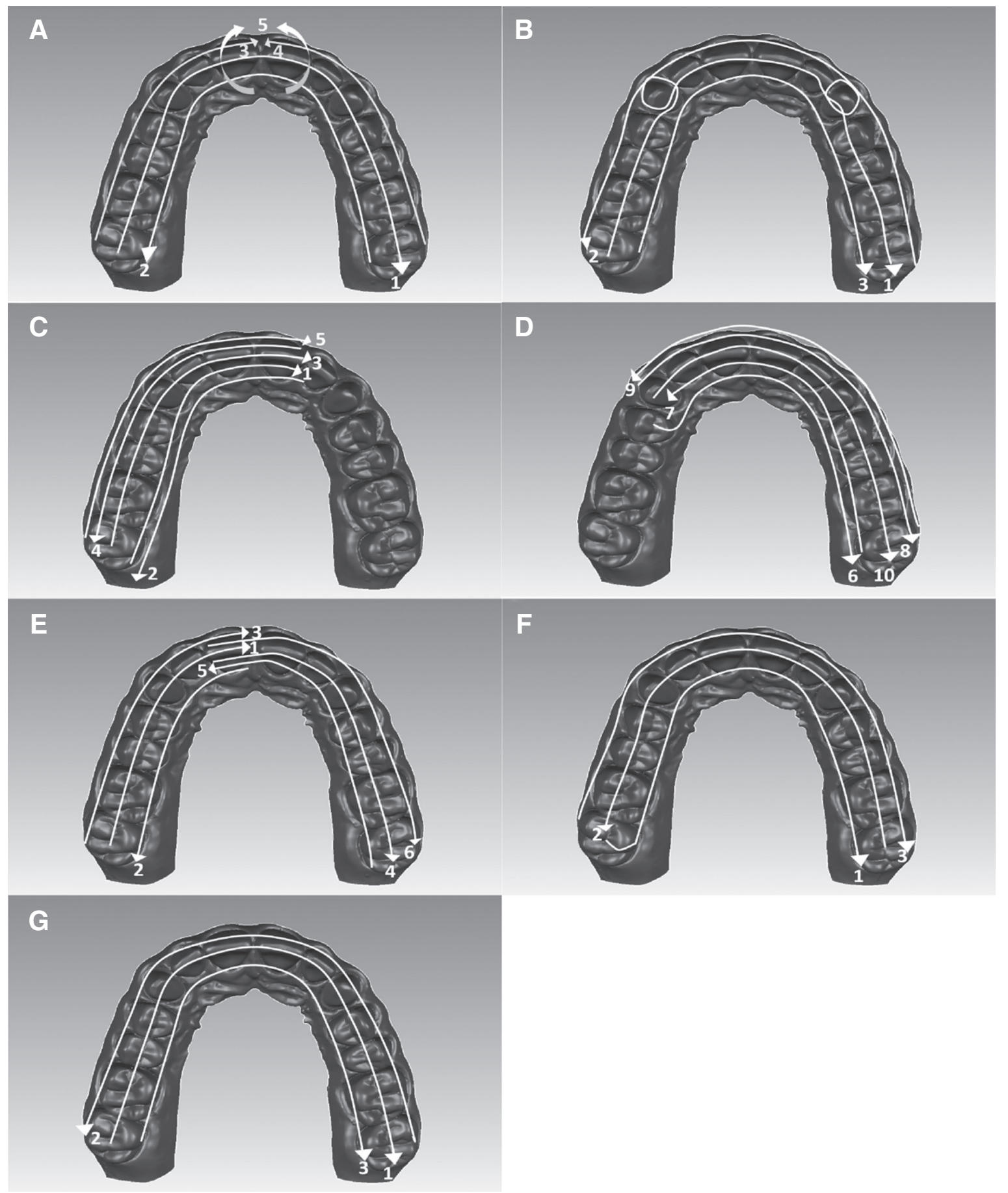

Fig. 1. Scan strategy for the ScanR. (A) iTero Element 2. The scan was started from the occlusal side of the right molars to the left occlusal side (1), continued the palatal side in the reverse direction (2), and toward the buccal side to the midline (3), moved to the opposite side and scanned to the midline (4), scanned by rolling over the anterior teeth (5). (B) Trios 3. Started from the occlusal side of the right molars to the left occlusal side by rolling over palatal, buccal, distal, and mesial side of preparations (1), continued the buccal side in the reverse direction (2), and toward the palatal side (3) (C) Omnicam, first half of arch. Started from the occlusal side of the right molars and tilted the scanner by $45^{\circ}$ in palatal direction (1), continued the palatal side $\left(90^{\circ}\right)$ from the left lateral to the right molar (2), toward the occlusal side in the reverse direction (3), scanned the buccal side with $45^{\circ}$ tilt (4) and $90^{\circ}$ tilt (5). (D) Omnicam, second half of arch.

Started from the occlusal side of the right premolar and continued the palatal side with $90^{\circ}(6)$ and $45^{\circ}$ (7) tilt, followed the buccal side with $45^{\circ}(8)$ and $90^{\circ}(9)$ tilt, and finally occlusal side (10). (E) Planmeca Emerald. Started from occlusal side of the right molar to the midline (1), continued the palatal side (2), and buccal side (3), scanned the second half similar to the first half, starting from the central tooth. (F) Primescan. Scanned the palatal side (1), occlusal side (2), and buccal side (3), respectively. (G) Virtuo Vivo. Scanned the occlusal side (1), buccal side (2), and palatal side (3), respectively (not defined a specific scan strategy by manufacturer). 

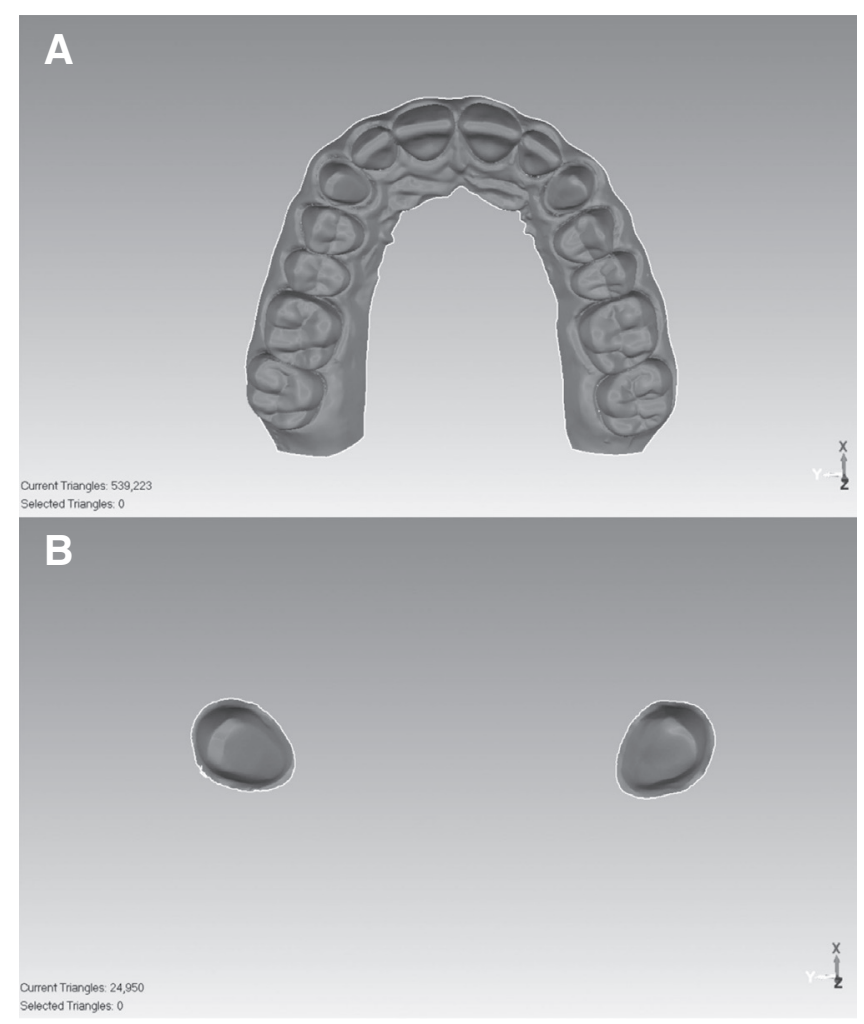

Fig. 2. The model scanned with the industrial reference scanner. (A) Complete arch, (B) Bilaterally prepared canine teeth digitally isolated from the complete arch.

ScanR 1 was determined as a reference scan and compared with the ScanR 2. Then the ScanR 2 was determined as a reference scan and was compared with the ScanR 1 . There were a total of 20 precision measurement combinations in each group. The necessity of performing the two-way pairwise comparison was mentioned because of the part comparison algorithm in the three dimensional software in a previous study. ${ }^{28}$

After the 3D comparative analysis, standard deviations and mean positive/ negative deviations were recorded in micrometers. For each IOS, the ScanR and ScanL values were recorded separately. The absolute mean deviations were obtained by calculating the arithmetic mean of the absolute values of the positive and negative deviations as mentioned by Treesh et al. ${ }^{28}$ Values for trueness and precision were calculated using absolute mean deviation values and standard deviation values, respectively.

All scan data were statistically analyzed to measure trueness and precision. The homogeneity and normality of distributions were tested by Kolmogorov-Smirnov and SkewnessKurtosis tests. The Kruskal Wallis test for non-normal distributed data and one-way ANOVA for normal distributed data were used. The Kruskal Wallis test was performed to compare the trueness differences between IOSs $(n=10)$. The pairwise comparisons were performed via the MannWhitney $\mathrm{U}$ test with a Bonferroni correction to control for the increased risk of Type I error by accounting for the number of comparisons. The Mann-Whitney $\mathrm{U}$ test was also used to compare differences among scanning sequences $(\mathrm{n}=5)$. One-way ANOVA and a post-hoc Tukey test were used to assess the precision differences between IOSs ( $\mathrm{n}=$ 20). The level of significance was set $\alpha=.05$ (except when the Bonferroni adjustment was used). After Bonferroni correction, the level of significance of $0.003(0.05 / 15)$ was considered. All statistical analysis was performed by using SPSS software (PASW Statistics 18.0, SPSS Inc., Chicago, IL, USA).

\section{RESULTS}

There were significant differences among IOSs for trueness and precision of digital impressions. The median trueness and mean precision values of each scanner and the $p$ values of the interaction between scanners for trueness and precision of single crown preparations are shown in Table 1 and Table 2. The statistically higher trueness was obtained from Primescan $(25 \mu \mathrm{m})$, followed by Trios $(40.5 \mu \mathrm{m})$, Omnicam $(41.5 \mu \mathrm{m})$, Virtuo Vivo $(52 \mu \mathrm{m})$, iTero $(70 \mu \mathrm{m})$, and Emerald $(73.5 \mu \mathrm{m})$ (Fig. 3). There was no statistically significant difference between Trios, Omnicam, Virtuo Vivo, and iTero $(P>.003)$. iTero did not exhibit statistically significant differences from Emerald and Virtuo Vivo for trueness. However, Emerald had statistically the lowest trueness among the all IOSs, except for iTero.

The highest precision was obtained from Primescan (10 $\pm 2 \mu \mathrm{m})$, followed by Trios $(11 \pm 3 \mu \mathrm{m})$, iTero $(12 \pm 3 \mu \mathrm{m})$, Omnicam $(18 \pm 5 \mu \mathrm{m})$, Virtuo Vivo $(37 \pm 19 \mu \mathrm{m})$, and Emerald $(60 \pm 27 \mu \mathrm{m})$. There was no statistically significant difference between Primescan, Trios, iTero, and Omnicam. The Emerald and Virtuo Vivo had statistically lower precision than Primescan, Trios, iTero, and Omnicam. Pairwise analysis revealed a statistically significant difference between Virtuo Vivo and Emerald.

Table 1. Trueness and precision values for each intraoral scanner

\begin{tabular}{llccr}
\hline & \multicolumn{2}{c}{$\begin{array}{c}\text { Trueness } \\
\text { First quartile }-\end{array}$} & Precision \\
& Median & $\begin{array}{c}\text { Third quartile } \\
(\text { Q1 - Q3) }\end{array}$ & Mean & $\begin{array}{r}\text { Standard } \\
\text { deviation }\end{array}$ \\
\hline Trios & 40.5 & $39-42$ & 11 & 3 \\
iTero & 70 & $45-87.25$ & 12 & 3 \\
Emerald & 73.5 & $64-76$ & 60 & 27 \\
Omnicam & 41.5 & $33.5-43.75$ & 18 & 5 \\
Primescan & 25 & $23.25-26.75$ & 10 & 2 \\
Virtuo Vivo & 52 & $44.75-55.25$ & 37 & 19 \\
\hline
\end{tabular}


Table 2. $P$ value results of Mann-Whitney $U$ test with Bonferroni correction for trueness and one-way ANOVA with post-hoc Tukey test for precision between intraoral scanners for single crown preparations

\begin{tabular}{|c|c|c|c|}
\hline Scanner & & Trueness & Precision \\
\hline \multirow[t]{5}{*}{ Trios } & iTero & .025 & .998 \\
\hline & Emerald & $.002^{\star}$ & $<.001^{\star \star}$ \\
\hline & Omnicam & 1 & .238 \\
\hline & Primescan & $<.001^{\star}$ & .999 \\
\hline & Virtuo Vivo & .017 & $<.001^{\star \star}$ \\
\hline \multirow[t]{5}{*}{ iTero } & Trios & .025 & .998 \\
\hline & Emerald & .705 & $<.001^{\star \star}$ \\
\hline & Omnicam & .010 & .471 \\
\hline & Primescan & $<.001^{\star}$ & .967 \\
\hline & Virtuo Vivo & .344 & $<.001^{* *}$ \\
\hline \multirow[t]{5}{*}{ Emerald } & Trios & $.002^{*}$ & $<.001^{\star \star}$ \\
\hline & iTero & .705 & $<.001^{\star *}$ \\
\hline & Omnicam & $.002^{\star}$ & $<.001^{\star \star}$ \\
\hline & Primescan & $<.001^{\star}$ & $<.001^{\star *}$ \\
\hline & Virtuo Vivo & $.002^{*}$ & $<.001^{* *}$ \\
\hline \multirow[t]{5}{*}{ Omnicam } & Trios & 1 & .238 \\
\hline & iTero & .010 & .471 \\
\hline & Emerald & $.002^{\star}$ & $<.001^{\star *}$ \\
\hline & Primescan & $<.001^{\star}$ & .102 \\
\hline & Virtuo Vivo & .009 & $<.001^{\star \star}$ \\
\hline \multirow[t]{5}{*}{ Primescan } & Trios & $<.001^{\star}$ & .999 \\
\hline & iTero & $<.001^{\star}$ & .967 \\
\hline & Emerald & $<.001^{\star}$ & $<.001^{\star \star}$ \\
\hline & Omnicam & $<.001^{\star}$ & .102 \\
\hline & Virtuo Vivo & $<.001^{\star}$ & $<.001^{\star \star}$ \\
\hline \multirow[t]{5}{*}{ Virtuo Vivo } & Trios & .017 & $<.001^{* *}$ \\
\hline & iTero & .344 & $<.001^{\star \star}$ \\
\hline & Emerald & $.002^{*}$ & $<.001^{* *}$ \\
\hline & Omnicam & .009 & $<.001^{* *}$ \\
\hline & Primescan & $<.001^{\star}$ & $<.001^{\star \star}$ \\
\hline
\end{tabular}

*Statistically significant difference among the scanners $(P<.003)$

** Statistically significant difference among the scanners $(P<.05)$

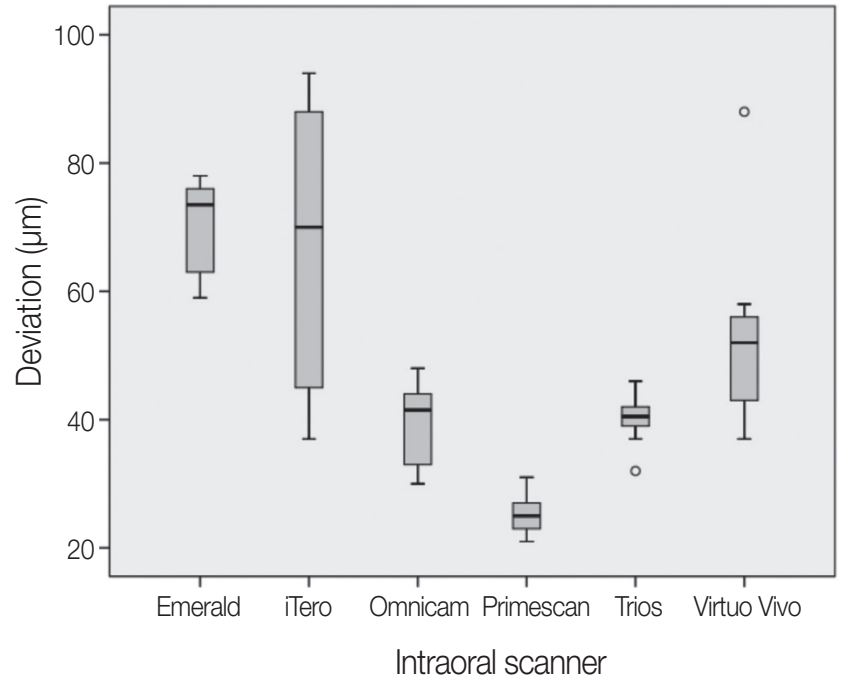

Fig. 3. Boxplot for trueness values of each intraoral scanner.

No significant difference was found between the precision values of the ScanR and ScanL obtained from each IOS for the prepared teeth. However, trueness was significantly affected by scanning sequence when using iTero. The ScanR had statistically lower trueness than the ScanL, for iTero $(P=.009)$. The trueness and precision values of the ScanR and ScanL for each IOS are shown in Table 3.

\section{DISCUSSION}

The aim of the present study was to evaluate the accuracy of single crown preparations using six IOSs, and to determine the effect of scanning sequence on accuracy. Based on the results of the present study, the first null hypothesis that there are no significant differences between digital impression accuracy of single crown preparations obtained by six IOSs was rejected. The second null hypothesis, that scan-

Table 3. Trueness and precision values of single crown preparations for Scan R and Scan L of each intraoral scanner (mean \pm standard deviation in micrometer)

\begin{tabular}{|c|c|c|c|c|c|c|}
\hline & \multicolumn{5}{|c|}{ Accuracy for the single crown preparations } & \multirow[b]{3}{*}{$P$ value } \\
\hline & \multicolumn{3}{|c|}{ Trueness } & \multicolumn{2}{|r|}{ Precision } & \\
\hline & Scan R & Scan L & $P$ value & Scan R & Scan L & \\
\hline Trios & $42 \pm 3$ & $38 \pm 4$ & .206 & $11 \pm 3$ & $11 \pm 3$ & 1 \\
\hline iTero & $74 \pm 4$ & $44 \pm 7$ & $.009^{*}$ & $10 \pm 2$ & $15 \pm 4$ & .995 \\
\hline Emerald & $67 \pm 21$ & $67 \pm 6$ & .114 & $59 \pm 30$ & $61 \pm 14$ & .727 \\
\hline Omnicam & $39 \pm 6$ & $42 \pm 7$ & .754 & $16 \pm 5$ & $21 \pm 6$ & .999 \\
\hline Primescan & $26 \pm 3$ & $24 \pm 3$ & .248 & $9 \pm 3$ & $11 \pm 2$ & 1 \\
\hline Virtuo Vivo & $44 \pm 8$ & $60 \pm 16$ & .059 & $43 \pm 14$ & $31 \pm 24$ & .381 \\
\hline
\end{tabular}

*Statistically significant difference $(P<.05)$ between Scan R and Scan L 
ning sequence does not affect accuracy, was partially rejected (for iTero).

The IOS accuracy varies with the quality of the point cloud obtained depending on the hardware and software algorithms. The mesh decimation algorithm regulates the total number of triangles by increasing the triangle density in curved surfaces and decreasing the triangle density on flatter surfaces. ${ }^{29}$ The size of the triangle varies depending on the scanning systems using different software algorithms. ${ }^{14}$ For this reason, different triangle configurations from the same surface may be obtained..$^{15}$ It is important to evaluate the accuracy of the IOSs using different hardware and software algorithms because these differences in triangles may affect accuracy. Therefore, the present study focused on the accuracy of new and actual intraoral scanners, and the software version of each scanner was specified. IOS accuracy can be evaluated directly through threedimensional comparisons of digital impressions, and indirectly through internal and marginal fit measurements of the fabricated restoration after scanning. The maximum clinically acceptable marginal gap was reported as $120 \mu \mathrm{m} .{ }^{4}$ Although a clinically acceptable deviation value for digital impressions is not clear in literature, their accuracy must be below $120 \mu \mathrm{m}$.

Several studies ${ }^{12,13,23,24}$ evaluated the accuracy of digital impressions obtained from the recently introduced Emerald, Virtuo Vivo, and Primescan IOSs. ${ }^{12,22}$ In these studies, edentulous or complete arch models with unprepared teeth were used as reference models. However, intraoral scanning of the prepared teeth is a commonly used and preferred procedure in daily clinical situations. This may be more complex than scanning unprepared teeth, because the prepared teeth involve various points which require high mesh density. ${ }^{30}$ For this reason, a reference complete arch model with right and left canine abutment teeth for single crowns was used in the present study. The commonly preferred Trios, iTero, and Omnicam IOSs, and the recently introduced Primescan, Virtuo Vivo, and Emerald IOSs were selected to evaluate the accuracy of the single crown preparations.

The recently introduced IOSs did not display similar accuracy. In the present study, Primescan showed the highest accuracy among all scanners tested, whereas Emerald had the lowest accuracy. However, the trueness of Virtuo Vivo was not statistically different from that of Omnicam, Trios, and iTero. Ender et al. ${ }^{12}$ reported that Primescan showed the highest trueness for digital impressions of the complete arch model with unprepared teeth, with statistically significant differences compared to iTero and Omnicam, but not Trios. The authors found that, similar to the results of the present study, Primescan had the highest trueness for the anterior segment. Latham et al. ${ }^{13}$ evaluated effect of scan strategy on complete arch scans using Cerec Omnicam, Planmeca Emerald, iTero Element, and Trios scanners. The accuracy of iTero, Emerald, and Trios were similar when four different scan strategies, guided by the various manufacturers' instructions, were used. The differences between those results and the ones found in the present study may relate to the use of different scan strategies. In the present study, the scan strategy described by its manufacturer was performed for each intraoral scanner. Another study ${ }^{23}$ reported that in an edentulous acrylic model with scan abutment, Emerald showed middle-low performance and Virtuo Vivo showed low performance when compared to Trios and Omnicam. Although the study models were different, results were similar to those found in the present study. Osnes et al. ${ }^{24}$ reported similar results in an edentulous model and stated that Emerald and Virtuo Vivo showed higher deviation than Trios and Omnicam.

Previous studies evaluating accuracy of single crown preparation reported that the accuracy varies depending on the IOSs utilized. ${ }^{8,911,18,19}$ In the present study, statistically significant differences were found between accuracy of six IOSs for single crown preparation. The most accurate scanner was Primescan (median trueness deviation $25 \mu \mathrm{m}$, mean precision deviation $10 \pm 2 \mu \mathrm{m})$, followed by Trios $(40.5 \mu \mathrm{m}$, $11 \pm 3 \mu \mathrm{m})$, Omnicam $(41.5 \mu \mathrm{m}, 18 \pm 5 \mu \mathrm{m})$, Virtuo Vivo $(52 \mu \mathrm{m}, 37 \pm 19 \mu \mathrm{m})$, iTero $(70 \mu \mathrm{m}, 12 \pm 3 \mu \mathrm{m})$, and Emerald $(73.5 \mu \mathrm{m}, 64 \pm 27 \mu \mathrm{m})$. Medina-Sotomayor et al. ${ }^{16}$ found Trios (trueness $9.7 \mu \mathrm{m}$, precision $15.1 \mu \mathrm{m}$ ) to be the most accurate scanner for a single tooth scan, when compared with iTero (trueness $11.2 \mu \mathrm{m}$, precision $18.4 \mu \mathrm{m}$ ) and Omnicam (trueness $20.6 \mu \mathrm{m}$, precision $33.2 \mu \mathrm{m}$ ). Another study ${ }^{11}$ showed that trueness of Trios was between $15.8 \pm$ $0.9 \mu \mathrm{m}$ and $20.6 \pm 1.0 \mu \mathrm{m}$, and precision was between 10.0 $\pm 2.1 \mu \mathrm{m}$ and $13.4 \pm 1.8 \mu \mathrm{m}$, depending on convergence angles of the preparation's mesial and distal walls. The root mean square values were statistically analyzed, and mean and standard deviation values for accuracy were presented. The numeric discrepancies in the results may be due to the use of different data types for statistical analysis and different reference model designs. In the studies of MedinaSotomayor et al. ${ }^{16}$ and Carbajal Mejía et al., ${ }^{11}$ a single prepared tooth (unattached to a model) or a model that also included onlay preparation and implant scan body were scanned, while single crown preparations on a complete arch were scanned in the present study. Thus, the distortion that occurs during a complete arch scan was taken into account when prepared teeth in different quadrants needed to be scanned. The trueness of IOS for single crown preparation was evaluated at four points determined by the sectional analysis method in an in vitro study. ${ }^{31}$ Although the evaluation methods and generation of IOSs used were different, Trios showed higher trueness than iTero, similar to the results of the present study. Boeddinghaus $e t a l .^{18}$ evaluated in vivo accuracy of single crown restorations via marginal fit of the copings. The Omnicam group showed statistically higher marginal gap than the Trios group. Contrary to this finding, in the present study, there was no statistically significant difference between accuracy of Omnicam and Trios. This may be related to the different study designs (in vivo and in vitro), potential processing errors during design and manufacture of the crown, and different version of IOS software. However, the study of Boeddinghaus $e t$ al. ${ }^{18}$ 
was standardized using the same workflow, design principles, software, material and milling machine to fabricate restorations and focused on IOS performance. Based on the different results obtained, the present study's authors have considered that IOSs may behave differently in in vivo and in vitro situations.

For each IOS, there is a scanning strategy specified by the manufacturer. However, manufacturer's instructions do not specify where scanning should begin, which is a concern especially in cases where both quadrants have prepared teeth. There may be differences in accuracy between the regions where scanning starts and ends; because cumulative errors may be seen with the stitching method as scanning moves from the starting point and continues towards proximal areas. ${ }^{15,27}$ Therefore, the effect of the scanning sequence on accuracy was evaluated in the present study. iTero had statistically significant differences based on scanning sequence (ScanR and ScanL) for accuracy of the prepared teeth. ScanL (44 $\pm 7 \mu \mathrm{m})$ showed higher trueness than ScanR $(74 \pm 4 \mu \mathrm{m})$. However, there were no statistically significant differences in precision of prepared teeth between ScanR and ScanL of IOSs. Anh et al. ${ }^{25}$ compared the precision of the digital impressions obtained by iTero and Trios. This study showed that iTero produced less precise digital impressions when scanning started from the right rather than from the left, similar to the trueness findings of the present study. Kim et al..$^{32}$ designed a multiple implant model and observed that iTero Element showed more deviation than Trios at the opposite side of the scans' starting region. Although different generations and softwares of iTero were used in the studies of Anh et al. ${ }^{25}$ and Kim et al., ${ }^{32}$ the region where scanning started affected the deviation in the opposite or complete arch. The result may be related to iTero's scan strategy, which merges left and right buccal scan data on the anterior region.

Like other in vitro studies evaluating IOS accuracy, the present study has some limitations. Intraoral factors such as saliva, limited mouth opening, patient movement, and different refractive indexes of teeth and gingiva were not considered. Further studies that evaluate the accuracy of newly introduced hardware and/or software of IOSs for single crown preparation under in vivo conditions are required. It will also be clinically useful to evaluate the IOS accuracy by comparing the marginal fit of the crown. The accuracy of actual and new IOSs should be evaluated on prepared teeth for long-span prostheses as well as for single crown preparation. In addition to the IOSs evaluated in the present study, the accuracy of IOSs recently introduced to the market, including Trios 4, Medit i500, and iTero Element 5D, should be evaluated.

\section{CONCLUSION}

Within the limitations of the present study, the accuracy of digital impressions for single crown preparations differed depending on the IOS and scanning sequence used. Primescan showed the highest trueness for single crown preparations. In terms of precision, Primescan, Trios, iTero, and Omnicam had better results than Emerald and Virtuo Vivo. Emerald showed the most deviation in trueness and precision. iTero resulted in more trueness deviation when scanning started from the right.

\section{ORCID}

Burcu Diker https://orcid.org/0000-0001-5367-9369

Önjen Tak https://orcid.org/0000-0002-3578-9078

\section{REFERENCES}

1. Memari Y, Mohajerfar M, Armin A, Kamalian F, Rezayani V, Beyabanaki E. Marginal adaptation of CAD/CAM all-ceramic crowns made by different impression methods: A literature review. J Prosthodont 2019;28:e536-44.

2. Ueda K, Beuer F, Stimmelmayr M, Erdelt K, Keul C, Güth JF. Fit of 4-unit FDPs from $\mathrm{CoCr}$ and zirconia after conventional and digital impressions. Clin Oral Investig 2016;20:283-9.

3. Chochlidakis KM, Papaspyridakos P, Geminiani A, Chen CJ, Feng IJ, Ercoli C. Digital versus conventional impressions for fixed prosthodontics: A systematic review and meta-analysis. J Prosthet Dent 2016;116:184-90.

4. Ahlholm P, Sipilä K, Vallittu P, Jakonen M, Kotiranta U. Digital versus conventional impressions in fixed prosthodontics: A review. J Prosthodont 2018;27:35-41.

5. Hayama H, Fueki K, Wadachi J, Wakabayashi N. Trueness and precision of digital impressions obtained using an intraoral scanner with different head size in the partially edentulous mandible. J Prosthodont Res 2018;62:347-52.

6. Yuzbasioglu E, Kurt H, Turunc R, Bilir H. Comparison of digital and conventional impression techniques: evaluation of patients' perception, treatment comfort, effectiveness and clinical outcomes. BMC Oral Health 2014;14:10.

7. ISO 5725-1. Accuracy (trueness and precision) of measurement methods and results - Part 1: General principles and definitions. International Standards Organization (ISO); Geneva; Switzerland, 1994. Available at: https://www.iso.org/ obp/ui/\#iso:std:iso:5725:-1:en. Accessed May 26, 2019.

8. Nedelcu RG, Persson AS. Scanning accuracy and precision in 4 intraoral scanners: an in vitro comparison based on 3-dimensional analysis. J Prosthet Dent 2014;112:1461-71.

9. Sim JY, Jang Y, Kim WC, Kim HY, Lee DH, Kim JH. Comparing the accuracy (trueness and precision) of models of fixed dental prostheses fabricated by digital and conventional workflows. J Prosthodont Res 2019;63:25-30.

10. Ender A, Mehl A. In-vitro evaluation of the accuracy of conventional and digital methods of obtaining full-arch dental impressions. Quintessence Int 2015;46:9-17.

11. Carbajal Mejía JB, Wakabayashi K, Nakamura T, Yatani H. Influence of abutment tooth geometry on the accuracy of conventional and digital methods of obtaining dental impressions. J Prosthet Dent 2017;118:392-9.

12. Ender A, Zimmermann M, Mehl A. Accuracy of completeand partial-arch impressions of actual intraoral scanning systems in vitro. Int J Comput Dent 2019;22:11-9. 
13. Latham J, Ludlow M, Mennito A, Kelly A, Evans Z, Renne W. Effect of scan pattern on complete-arch scans with 4 digital scanners. J Prosthet Dent 2020;123:85-95.

14. Ender A, Zimmermann M, Attin T, Mehl A. In vivo precision of conventional and digital methods for obtaining quadrant dental impressions. Clin Oral Investig. 2016;20:1495-504.

15. Zimmermann M, Koller C, Rumetsch M, Ender A, Mehl A. Precision of guided scanning procedures for full-arch digital impressions in vivo. J Orofac Orthop 2017;78:466-71.

16. Medina-Sotomayor P, Pascual-Moscardo A, Camps A I. Accuracy of 4 digital scanning systems on prepared teeth digitally isolated from a complete dental arch. J Prosthet Dent 2019;121:811-20.

17. Rauch A, Reich S, Schierz O. Chair-side generated posterior monolithic lithium disilicate crowns: clinical survival after 6 years. Clin Oral Investig 2017;21:2083-9.

18. Boeddinghaus M, Breloer ES, Rehmann P, Wöstmann B. Accuracy of single-tooth restorations based on intraoral digital and conventional impressions in patients. Clin Oral Investig 2015;19:2027-34.

19. Hack GD, Patzelt SBM. Evaluation of the accuracy of six intraoral scanning devices: an in-vitro investigation. Am Dent Assoc 2015;10:1-5.

20. Mühlemann S, Greter EA, Park JM, Hämmerle CHF, Thoma DS. Precision of digital implant models compared to conventional implant models for posterior single implant crowns: A within-subject comparison. Clin Oral Implants Res 2018;29:9316.

21. Kim RJ, Park JM, Shim JS. Accuracy of 9 intraoral scanners for complete-arch image acquisition: A qualitative and quantitative evaluation. J Prosthet Dent 2018;120:895-903.

22. Haddadi Y, Bahrami G, Isidor F. Effect of software version on the accuracy of an intraoral scanning device. Int J Prosthodont 2018;31:375-6.

23. Di Fiore A, Meneghello R, Graiff L, Savio G, Vigolo P, Monaco C, Stellini E. Full arch digital scanning systems performances for implant-supported fixed dental prostheses: a comparative study of 8 intraoral scanners. J Prosthodont Res 2019;63:396403.

24. Osnes CA, Wu JH, Venezia P, Ferrari M, Keeling AJ. Full arch precision of six intraoral scanners in vitro. J Prosthodont Res 2020;64:6-11.

25. Anh JW, Park JM, Chun YS, Kim M, Kim M. A comparison of the precision of three-dimensional images acquired by 2 digital intraoral scanners: effects of tooth irregularity and scanning direction. Korean J Orthod 2016;46:3-12.

26. GOM: ATOS Core - Features: https://www.atos-core.com/ en/features.php\#3dScanning. Accessed 23/9/19.

27. Kim JE, Amelya A, Shin Y, Shim JS. Accuracy of intraoral digital impressions using an artificial landmark. J Prosthet Dent 2017;117:755-61.

28. Treesh JC, Liacouras PC, Taft RM, Brooks DI, Raiciulescu S, Ellert DO, Grant GT, Ye L. Complete-arch accuracy of intraoral scanners. J Prosthet Dent 2018;120:382-8.

29. Mandelli F, Gherlone E, Gastaldi G, Ferrari M. Evaluation of the accuracy of extraoral laboratory scanners with a singletooth abutment model: A 3D analysis. J Prosthodont Res
2017;61:363-70.

30. Richert R, Goujat A, Venet L, Viguie G, Viennot S, Robinson P, Farges JC, Fages M, Ducret M. Intraoral scanner technologies: A review to make a successful impression. J Healthc Eng 2017;2017:8427595.

31. Park JM. Comparative analysis on reproducibility among 5 intraoral scanners: sectional analysis according to restoration type and preparation outline form. J Adv Prosthodont 2016; 8:354-62.

32. Kim RJ, Benic GI, Park JM. Trueness of digital intraoral impression in reproducing multiple implant position. PLoS One 2019;14:e0222070. 